\title{
The TeleKyb Framework for a Modular and Extendible ROS-based Quadrotor Control
}

\author{
Volker Grabe, Martin Riedel, Heinrich H. Bülthoff, Paolo Robuffo Giordano, Antonio Franchi
}

\begin{abstract}
The free and open source Tele-Operation Platform of the MPI for Biological Cybernetics (TeleKyb) is an endto-end software framework for the development of bilateral teleoperation systems between human interfaces (e.g., haptic force feedback devices or gamepads) and groups of quadrotor Unmanned Aerial Vehicles (UAVs). Among drivers for devices and robots from various hardware manufactures, TeleKyb provides a high-level closed-loop robotic controller for mobile robots that can be extended dynamically with modules for state estimation, trajectory planning, processing, and tracking. Since all internal communication is based on the Robot Operating System (ROS), TeleKyb can be easily extended to meet future needs. The capabilities of the overall framework are demonstrated in both an experimental validation of the controller for an individual quadrotor and a complex experimental setup involving bilateral human-robot interaction and shared formation control of multiple UAVs.
\end{abstract}

\section{INTRODUCTION}

The challenges inherent to software development for a robotic platform have constantly changed over the last decade. Initially, the program code for robotic platforms comprising a certain set of sensors, actuators and controllers were developed solely for a specific hardware architecture, making the reuse of software complicated and thus often impractical. Recently, the design of robotic platforms has moved to setups that are more modular and allow for a simplified integration or exchange of individual hardware components [1]. Therefore the underlying software architectures that operate these robots required a fundamental paradigm shift consisting of, e.g., the introduction of increasing levels of abstractions.

Since then, robotic middleware solutions migrated to a thin-design paradigm that supports the development of modular components and increases the ability to reuse existing code. Several frameworks follow this new paradigm (see, e.g., [2], [3]) with the Robot Operating System (ROS) being one of the most popular [4]. Since its release in 2007, several hundred packages have been published.

Despite the clear benefits that ROS introduced to the robotic community, the concept of ROS is not particularly new, but it is actually comparable to existing solutions such

V. Grabe, M. Riedel, H. H. Bülthoff and A. Franchi are with the Max Planck Institute for Biological Cybernetics, Spemannstraße 38, 72076 Tübingen, Germany. E-Mail: \{volker.grabe, martin.riedel, hhb, antonio.franchi\}@tuebingen.mpg.de.

P. Robuffo Giordano is with the CNRS at IRISA, Campus de Beaulieu, 35042 Rennes Cedex, France prgeirisa.fr.

V. Grabe is additionally with the University of Zurich, Andreasstr. 15, 7050 Zurich, Switzerland. H. H. Bülthoff is additionally with the Department of Brain and Cognitive Engineering, Korea University, Seoul, 136-713 Korea. as, e.g., the Inter Process Communication (IPC) library [5]. That is, ROS provides mainly a communication interface between independent pieces of a framework together with a platform to share released code. In order to implement their algorithms on a particular robotic platform, roboticists are still forced to develop the appropriate drivers and controllers and to link them into a reliable framework. While drivers for several hardware components have been gratefully shared by other scientists through ROS, the development of an appropriate control framework still remains a challenging and time-consuming task, for example in the popular research field on Unmanned Aerial Vehicles (UAVs).

Over the last years, use of small quadrotor UAV platforms has gained immense popularity in the robotics community due to their small costs, robustness, flexibility, and hovering capabilities. However, at the best of our knowledge, only few research labs publicly released their complete quadrotor control frameworks. Moreover, each framework is typically limited to a particular chain constituted by a specific control device, a specific state estimator, and specific flight controller and vehicle driver. For the commercially available AR.Drone $^{1}$, a position controller has been released [6]: this is based on the velocity controller provided by the manufacturer. However, this solution is highly specific to quadrotor platforms that are endowed with an on-board velocity sensor and accept velocity commands. Similarly, a control framework restricted to quadrotors from Ascending Technologies $^{2}$ has been published [7]. Both frameworks include an estimator of the the quadrotor state that exploits a low-frequency visual-based pose estimation together with acceleration and angular velocity readings from the on-board inertial measurement unit (IMU).

However, both systems lack the support for other hardware platforms, are limited to a single mode of operation, and do not support the simultaneous control of a swarm of quadrotors for cooperative operations. Furthermore, they have not been designed to facilitate input modalities other than the specification of predefined waypoints and thus do not allow for a bilateral control in telepresence situations using, e.g., force feedback devices as in [8].

In order to compensate for this lack of basic features, in this paper we propose a complete modular control framework for a generic quadrotor UAV based on ROS. By providing structured interfaces, the user can dynamically extend the framework, while still maintaining basic security

\footnotetext{
${ }^{1}$ http: //ardrone2.parrot.com
}

${ }^{2}$ http: //asctec.de 
mechanisms, like state supervision and fall-back modules. The full control chain released to the public is completely extendable and already includes support for various input devices, multiple heterogeneous UAVs, and different state estimators.

The remainder of this paper is structured as follows. The main components and interfaces of the proposed framework are discussed in Sec. II. In Section III, all components involved with the actual control of the quadrotor UAVs are discussed in more detail and we summarize the modules included in the open source code release of this project in Sec. IV. Finally, we present and discuss our experimental evaluation in Sec. V and VI before the paper is concluded in Sec. VII.

\section{Major Components of TeleKyb}

TeleKyb is an extensive software framework consisting of more than 50 ROS packages for the end-to-end solution of quadrotor based setups. This includes a human interface layer for direct modes of interaction with the human operator, a control layer for motion planning and actuation of the quadrotor, and a hardware interface layer used to interface the particular quadrotor hardware. In the following, we describe the main components of TeleKyb as illustrated in Fig. 1.

\section{A. Human Interface}

Human input appliances that feed back haptic cues to the operator have become a popular field of research in the human-robot interaction community [9]. Since the variety of haptic-device manufacturers all use different SDKs for their hardware, TeleKyb Haptics provides a unique interface for the common types of haptic devices and thus implements the haptic control algorithm using standardized methods. These methods are then implemented by the specific hardware driver. Currently, TeleKyb supports all device classes of Force Dimension ${ }^{3}$.

Human input devices which do not provide haptic feedback, such as common joysticks and gamepads, can be directly used with TeleKyb if they are natively supported by the operating system.

\section{B. TeleKyb Base}

TeleKyb Base provides a variety of helper classes which are aimed to support the roboticists in the development of control algorithms. Among an improved memory management and multiple conversion functions for various purposes, the ROS parameter system has been greatly extended.

TeleKyb Options provide a mechanism to expose parameters of any node to external entities which can be updated at run-time. Thus, they combine the capabilities of the default ROS parameters and the ROS dynamic reconfigure ${ }^{4}$ package. However, as opposed to the original ROS parameters, the Option class supports optional bounds, read-only options, handles the correct namespace automatically and is not

\footnotetext{
${ }^{3}$ http://forcedimension.com/

${ }^{4}$ http://ros.org/wiki/dynamic_reconfigure
}

restricted to the native datatypes integer, double, and string. For example, it is possible to handle matrices or vectors as parameters.

\section{TeleKyb Core}

TeleKyb Core is a high-level closed-loop robotic controller composed of four main elements with distinct functionalities. The State Estimator estimates the current pose of the robot, the Trajectory Behavior unit computes the next desired position and velocity, the Trajectory Processor implements additional functionality such as obstacle avoidance, and the Trajectory Tracker computes the next commands which are then sent to the robot as explained in more detail in Sec. III.

\section{ROS-Simulink Bridge}

MATLAB/Simulink is a popular tool for the simulation and control of dynamic systems, including robots. However, depending on the release, it links against older versions of certain libraries (e.g., boost) than those present on current unix operating systems and which are therefore used when TeleKyb is built. To the best of our knowledge, all the currently available ROS-MATLAB interfaces provide communication between MATLAB and other ROS nodes via relatively slow network transmission rather than a native integration.

To overcome this limitation, we setup an environment to compile ROS against specific MATLAB versions and operating systems. The resulting shared libraries are then linked against MATLAB S-functions to implement the Simulink side of the ROS interface. Thanks to this setup, generic S-function publisher and subscriber blocks use the native ROS communication stack for the exchange of message with other nodes. We have recently joined efforts with MathWorks to integrate this ROS interface into coming versions of MATLAB.

Within TeleKyb, this allows for the use of MATLAB/Simulink on top of the TeleKyb Core and Simulink can be utilized for the implementation and evaluation of higher-level algorithms or alternative controllers. Similarly, the ROS-MATLAB bridge can be utilized for the integration of human input devices with existing ROS drivers into MATLAB programs. Our bridge has already been successfully applied in various setups [10], [11].

\section{E. Obstacle Provider}

Applications for mobile robots often include the necessity to detect and dynamically react to obstacles in the environment. For this purpose, TeleKyb uses an Obstacle Provider which maintains a list of obstacle modules. TeleKyb provides modules for the definition of constant obstacles, fixed geometric structures, and dynamically moving obstacles provided from either on-board observations or external sources such as motion tracking systems.

\section{F. Robot Interface}

TeleKyb is in general not restricted to be only used with quadrotors UAVs, but can rather be employed with a 


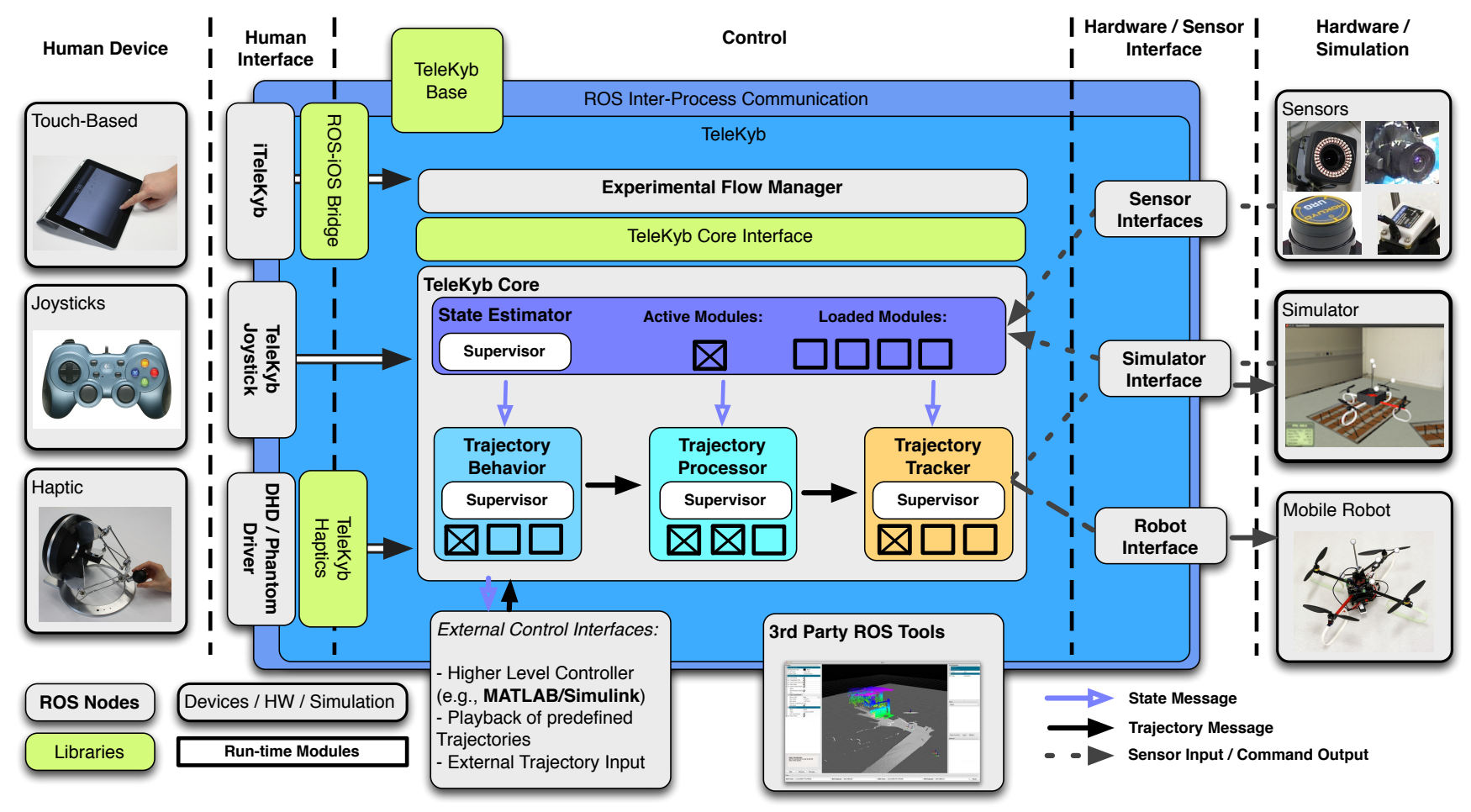

Fig. 1: High-level overview of major TeleKyb components. TeleKyb bridges the gap between user interfaces and robot hardware by introducing the TeleKyb interface layer.

wide variety of mobile robots since the implementation of hardware interfaces is strictly separated from the underlying algorithms. For each vehicle, suitable controller modules (see Sec. III) have to be provided together with an implementation of the Robot interface. This interface allows for both high frequency messages (such as control commands and sensor data) and asynchronous communication that can be used, e.g., to update controller gains or trigger an emergency procedure. Incoming data from the robot can be examined to react automatically on certain events such as low battery or detection of unexpected changes in some variables of the system. All communication can be exposed to ROS topics for monitoring or visualization purposes.

\section{G. Simulator Interface}

Robotic simulators provide both the simulation of a particular robotic hardware and the environment surrounding the robot. For the use of TeleKyb with a simulation, both a robot interface and a state estimation module (as introduced in Sec III-A) have to be provided. Currently, TeleKyb provides full supports for SwarmSimX [12], while an integration of $\mathrm{v}$-rep ${ }^{5}$ is under active development.

\section{TRAJectory Processing AND CONTROL}

In this section, the details of the TeleKyb Core and the different stages of our universal quadrotor controller are explained.

\footnotetext{
${ }^{5}$ http: //coppeliarobotics.com
}

\section{A. State Estimation}

The State Estimator consists of a Supervisor and a number of run-time loadable modules that provide the actual state estimation functionality. While the Supervisor is responsible for loading, unloading, and transition between the individual estimator modules, the currently active module generates a standardized state message $(p, \dot{p}, q, \omega)$, where $p, \dot{p}, q, \omega$ denote the position, velocity, orientation and angular velocity of the robot, respectively. This message is then distributed to the other parts of the controller within the TeleKyb Core. The active state estimation module can be changed at runtime, which allows for a safe fall-back state estimator module once another, e.g. vision based, estimator becomes too noisy or returns unreliable data. Also, estimators based on different sensors can be used for different behaviors of the flight, e.g., during the take-off or cruse phase, as described in Sec. III-B.

For external optical tracking systems, TeleKyb supports all systems that implement the free and open VRPN standard. In particular, this is the case with both the Vicon ${ }^{6}$ and the OptiTrack ${ }^{7}$ tracking systems. Since the state message requires the additional knowledge of the linear and angular velocities $\dot{p}$ and $\omega$, they are numerically computed from the pose $p$ and the orientation $q$ as obtained from the tracking systems using the signal filtering libraries provided with TeleKyb.

\footnotetext{
${ }^{6}$ http://vicon.com

${ }^{7}$ http: //naturalpoint. com/optitrack
} 


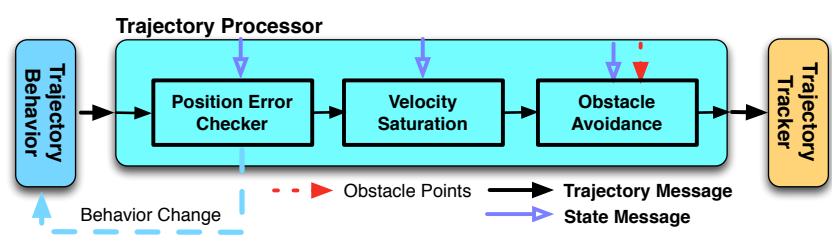

Fig. 2: Priority list of Trajectory Processor modules as they dynamically alter the current trajectory message before it is executed.

\section{B. The Behavior System}

The Trajectory Behavior system is one of the most powerful components of the control framework and contributes largely to its flexibility. Similar to the State Estimator, a Supervisor manages individual modules which provide the actual functionality, in the following referred to as Behaviors. The active behavior is responsible for the generation of a trajectory message every time a new state was made available by the State Estimator.

Behaviors dynamically generate trajectories for any given task during a robotic experiment. While many default behaviors such as Take-Off, Hover, Fly-To, Trajectory Following, Human Control and Land are already included with TeleKyb, the user can easily add new behaviors by implementing a provided interface. Consequently, the Supervisor can switch between individual behaviors in order to describe higherlevel motions such as Take-Off $\rightarrow$ Brake $\rightarrow$ Hover $\rightarrow$ Land . Therefore, the behavior system is equivalent to a finitestate machine with exactly one active behavior at any time and well-defined rules for the transitions. To ensure safe transitions, a type is assigned to each behavior and transitions are only allowed between behaviors of certain types, e.g., Ground $\rightarrow$ Take-Off, Air $\rightarrow$ Air, .... Additionally, they can be linked by defining a pointer to the next behavior. This behavior will then become active once the previous one terminates. This termination criterion can be either defined within each behavior or triggered by user input. A behavior without a follow-up element will be automatically followed by the Brake behavior which in turn will be followed by the Hover behavior until a new behavior is requested.

Since behaviors can be chained and each sequence forms a more complex behavior again, this simple design principle allows to accommodate a wide range of experimental conditions.

\section{The Trajectory Processor}

The Trajectory Processor maintains multiple modules able to dynamically alter the trajectory message generated from the active Behavior before it is passed on to the Trajectory Tracker. Several modules can be active at the same time and are applied to each trajectory message in sequential order, as shown in Fig. 2.

Generally, trajectory processor modules can either alter the trajectory message directly, e.g., to implement obstacle avoidance, or influence the system in a more indirect way by initiating a Behavior switch, e.g., to brake if some unexpected conditions were detected. Furthermore, modules can observe and interact with other components of the TeleKyb Core through the option system and, e.g., change the gains of the controller to less aggressive settings.

\section{The Trajectory Tracker}

The Trajectory Tracker computes the needed robot commands from both the current state and trajectory message.

The control design is based on the natural decoupling between attitude and linear dynamics (the former is independent from the latter). The design is then made in two steps in a classical cascade fashion: first, a fast attitude controller enforces tracking of a desired attitude unit quaternion $q_{d e s}(t)$ to the current quadrotor orientation $q(t)$ by exploiting the full actuated rotational dynamics for quadrotor UAVs (three available torque inputs). Then, a slower position controller generates the attitude set-point $q_{d e s}(t)$ needed to properly reorient the direction of the fourth input (thrust) so as to obtain the desired translational motion, i.e., for eventually letting the position $p(t)$ to track a desired reference $p_{\text {des }}(t)$. Compared to other control designs (e.g, feedback linearization), this solution can result in a less performant transient behavior but benefits from a higher robustness w.r.t. disturbances, parametric and modeling uncertainties thanks to the aforementioned dynamical separation.

\section{E. The on-board Low-Level Controller}

While TeleKyb supports UAVs from Ascending Technologies, KMel Robotics ${ }^{8}$, and MikroKopter ${ }^{9}$, the integration of quadrotors from MikroKopter is currently most advanced due the possibility to implement the on-board low-level controller manually.

The on-board low-level controller is in charge of letting the quadrotor orientation $q(t)$ track a desired reference $q_{d e s}(t)$. As classically done, this is achieved by neglecting the couplings among the three body axes, and by treating each individual rotation (roll, pitch, yaw) as a separate channel modeled as a double integrator with input the correspondent body torque. The adopted controller is then a simple PID with saturated integral term in order to prevent wind-up issues. Readings from the on-board gyros are exploited as velocity feedback, while a complementary filter provides estimates of the UAV attitude by fusing together accelerometer and gyro readings from the IMU.

\section{F. TeleKyb Core Interface}

The TeleKyb Core Interface allows to dynamically load and configure the Estimation, Behavior, Processing and Tracking modules. Furthermore, it provides automatic callbacks to track certain events such as a behavior transition in the Trajectory Behavior stage.

\section{G. Experimental Flow Manager}

The Experimental Flow Manager controls the flow of an entire experiment consisting of one or more TeleKyb Cores, one for each robot. For this purpose, it can be launched from

\footnotetext{
${ }^{8}$ http: // kmelrobotics.com

${ }^{9}$ http://mikrokopter.de
} 
TABLE I: Hardware supported with TeleKyb download

\begin{tabular}{|l|l|}
\hline Input Devices & Gamepads, Devices from Force-Dimension \\
\hline Tracking Systems & Vicon, OptiTrack, and others with VRPN support \\
\hline UAVs & $\begin{array}{l}\text { MikroKopter (with provided extended low-level } \\
\text { firmware), AscTec UAVs (beta version only) }\end{array}$ \\
\hline
\end{tabular}

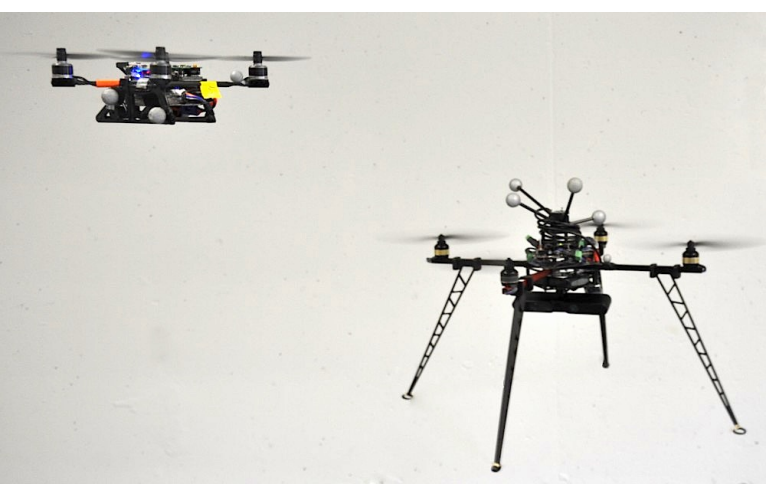

Fig. 3: The quadrotors used for the evaluation: Nano+ from kmel (left) and a MK-Quadro from MikroKopter (right).

anywhere in the network. Using the TeleKyb Core Interface, the TeleKyb Cores can be configured and manipulated online, e.g., to specify when Behaviors become active and how they are configured. In this role, the Experimental Flow Managers can be used to trigger events on the TeleKyb Cores, for example when requested by input devices connected to the network. Additionally, the Experimental Flow Manager can be informed by a TeleKyb Core to react on events such as the termination of a Behavior. This concept was also used to implement control through a touch-based hand-held device.

\section{Source Code Release}

We released all major components of TeleKyb under the BSD license. A current version of TeleKyb can be obtained from https://svn.tuebingen.mpg.de/kyb-robotics/ TeleKyb/trunk. Table I lists the hardware drivers provided with TeleKyb. A documentation including quick start instructions has been integrated into the ROS Wiki at http://ros.org/wiki/telekyb. For license reasons, we were not allowed to provide the communication interface for the vehicles from KMel Robotics. Please note that, according to the BSD license, the software is provided 'as is' and we cannot be hold responsible for any damage or harm caused by the use of our software.

\section{EXPERIMENTS}

In order to both evaluate the accuracy of the controller included with TeleKyb, and the ability to design complex experiments, we conducted two individual sets of experiments described in Sec. V-A and Sec. V-B, respectively.

As for the hardware setup, we used two different types of quadrotors, a Nano+ from KMel Robotics with a diameter of $0.19 \mathrm{~m}$ and a weight of $0.230 \mathrm{~kg}$, and a $M K$-Quadro from MikroKopter with a diameter of $0.50 \mathrm{~m}$ and a weight of $1.050 \mathrm{~kg}$. Both are shown in Fig. 3.
The state of the robot was retrieved via an external motion capture system at the frequency of $120 \mathrm{~Hz}$ and submillimeter precision. The desired roll, pitch, yaw-rate, and thrust commands computed by the controller are sent to attitude controller implemented on the micro controller by means of a wireless serial communication.

Both UAVs were flown using the same controller gains. A mass estimator included in TeleKyb estimated the actual quadrotor mass starting from a rough initial estimate: this allowed to autonomously compensate for the different characteristics of the two vehicles. The velocity was restricted to $1.0 \mathrm{~m} / \mathrm{s}$ through configurable options for safety reasons. Likewise, the maximum tilt angle was limited to $20^{\circ}$, thus also limiting the maximum achievable linear acceleration.

\section{A. Experiment for Evaluation of the Controller}

The first set of experiments was designed to evaluate the TeleKyb controller. In an initial setup, we aimed to test the Trajectory Tracker which provides appropriate orientation (i.e., attitude) and thrust commands to the UAVs given the desired positions, velocities, and accelerations at each control iteration. Both UAVs were flown along the same trajectory (a 'eight' shape) with a given velocity profile defined prior to the experiment. One loop along whole curve lasted $10 \mathrm{~s}$ with a peak velocity of $0.65 \mathrm{~m} / \mathrm{s}$.

In a second experiment, the whole controller chain, including a Fly-To Behavior that computes the trajectory towards a defined target on-line, was investigated. Both UAVs were commanded to fly to a target location approximately $2 \mathrm{~m}$ from the hovering UAV and stop there.

\section{B. Experiment with a Swarm and Human In-the-loop}

To demonstrate the capabilities of the TeleKyb framework with respect to more complex scenarios including presence of multiple UAVs, obstacle avoidance, human-robot interaction and haptic control, we designed an experimental setup with four MK-Quadro UAVs implementing various Behaviors and Processor Modules.

The following ROS nodes are running during the experiment: 4 MikroKopter Interfaces, 4 TeleKyb Cores, 4 Obstacle Providers providing dynamic and static obstacle boundaries, 1 VRPN tracking node, $1 \mathrm{x}$ haptic device node, and 1 Experimental Flow Manager which loads and configures all Behaviors and reacts to user input and dynamic callbacks from the TeleKyb Cores.

\section{Results AND Discussion}

\section{A. Experiment for Evaluation of the Controller}

In Figure 5, the results of the first set of experiments are demonstrated. The desired trajectory was compared to the achieved trajectory for both vehicles. For the heavy MKQuadro, we measured an average position error of $0.034 \mathrm{~m}$ and an average velocity error of $0.042 \mathrm{~m} / \mathrm{s}$. The error did not exceed $0.062 \mathrm{~m}$ and $0.104 \mathrm{~m} / \mathrm{s}$, respectively. For the lighter Nano+, we found smaller average position errors of $0.021 \mathrm{~m}$ which did not exceed $0.039 \mathrm{~m}$. The velocity error was in average $0.026 \mathrm{~m} / \mathrm{s}$ and always less than $0.058 \mathrm{~m} / \mathrm{s}$ for the 


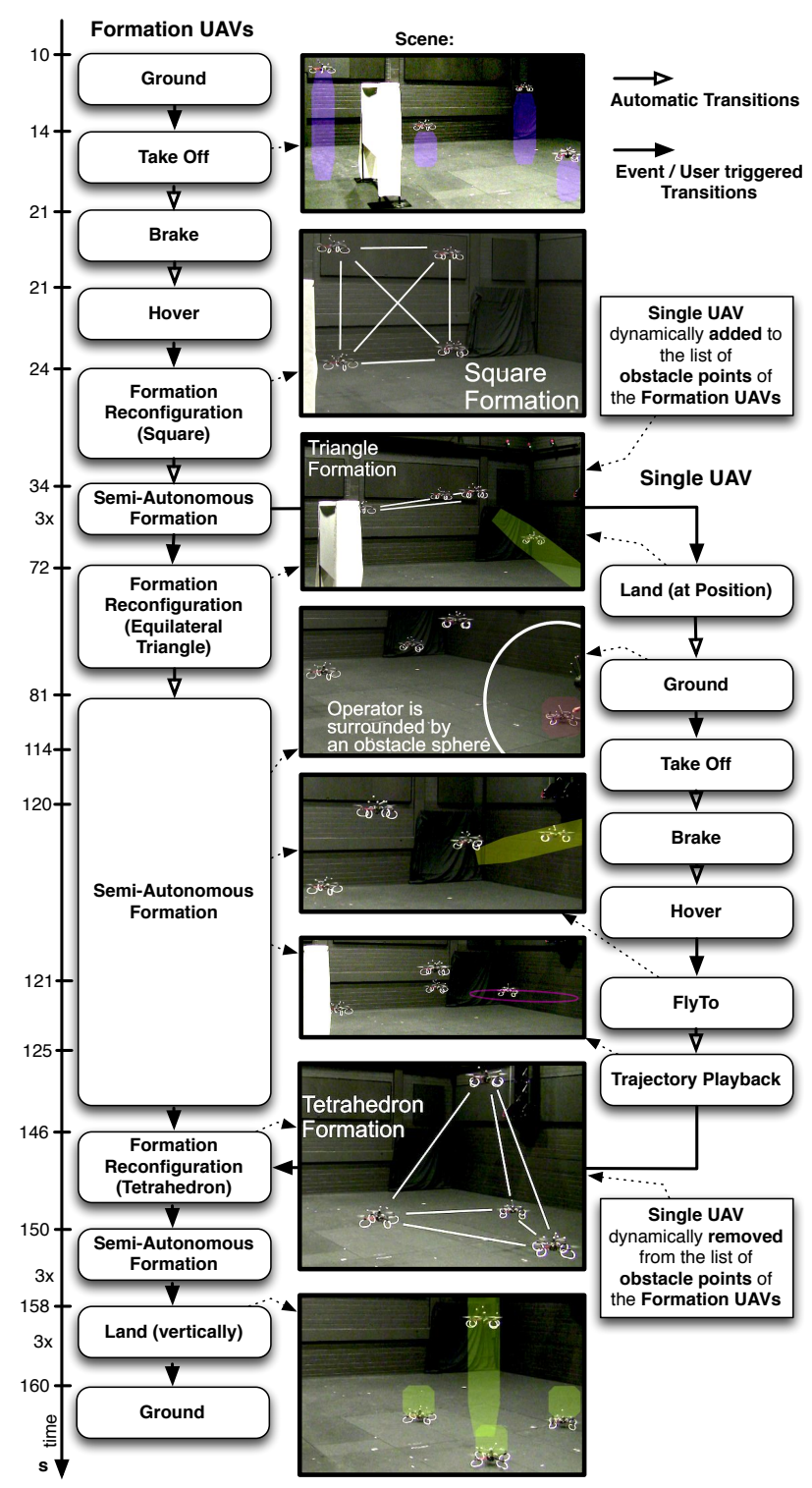

Fig. 4: Experimental flow during the human controlled swarm experiment as described in Sec. V-B. The left and right column list the currently active Behavior for the quadrotors in the swarm and the individual vehicle respectively. The time line is aligned to the attached video, also accessible at http://antoniofranchi.com/videos/telekyb.html (see Sec. VI-B).

Nano+ when ignoring an outlier caused by the tracking system at time $3 \mathrm{sec}$.

The results for the flight to a distant target are shown in Fig. 6. Both UAVs received the command after 0.3 seconds and accelerated up to their predefined maximum velocity. The heavier MK-Quadro started deceleration shortly before the Nano+ in order to stop and hover above the assigned goal location. Both vehicles stopped within $0.02 \mathrm{~m}$ of the target.

\section{B. Experiment with a Swarm and Human In-the-loop}

The experiment of the more complex swarm flight demonstrated the capabilities of the behavior based architecture.

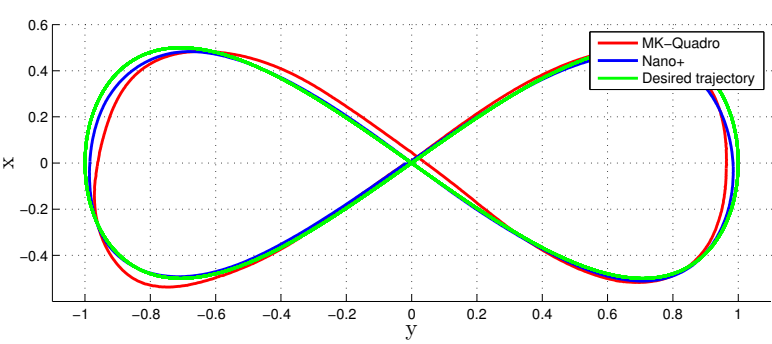

(a)

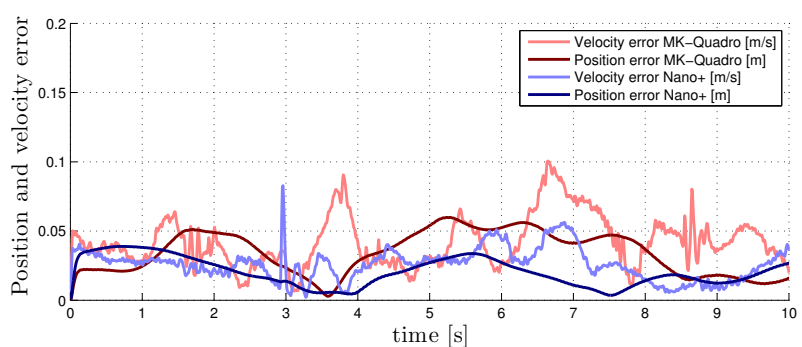

(b)

Fig. 5: Trajectory of the Nano+ and the MK-Quadro along a eight shaped against the desired trajectory. (a) Position of the quadrotors together with the desired trajectory and (b) Norm of the position and velocity tracking errors during one traversal of the trajectory for both vehicles.

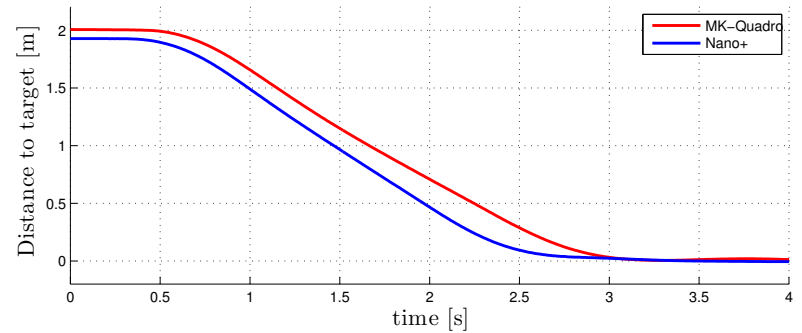

Fig. 6: Distance to the target for a Nano+ and an MK-Quadro in a sidestep maneuver. Both velocity and acceleration were saturated for safety reasons as evident from the plots.

Fig. 4 depicts the experimental flow during this demonstration. Additionally, a descriptive video of this experiment is attached to this submission and can also be accessed at http://antoniofranchi.com/videos/telekyb.html.

At multiple times (e.g., at 24, 34, 72, 81, 146, $150 \mathrm{sec}$ ) the 4 UAVs switched from or to a formation which required an exchange of some information among the robots such as current poses and inter-distances of neighboring UAVs. Note that formation control was implemented in a completely decentralized way, thus not requiring the Experimental Flow Manager or any other centralized controller.

At second 34, a single UAV left the formation and the remaining UAVs rearranged into a equilateral triangle. Simultaneously, the Experimental Flow Manager reconfigured the Obstacle Avoidance Processor Module of the remaining swarm to consider the single UAV as an obstacle. At second 81 , this UAV landed at a predefined position to undergo a simulated maintenance, while the remaining UAVs kept being in control of the human operator. After having taken 
off again, the single UAV entered a circular trajectory until it rejoined the formation at second 146 upon user command. After $160 \mathrm{sec}$, all UAVs landed vertically.

\section{CONCLUSION}

In this paper, we introduced a control framework for quadrotor UAVs called TeleKyb. We demonstrated that TeleKyb presents a complete end-to-end solution containing all the necessary components from drivers for human input devices to hardware support for quadrotors from different manufacturers. Complex experimental setups for high-level robotic tasks such as exploration or mapping can be created without in-depth knowledge of control theory. Nevertheless, by relying on ROS for inter process communication and well defined interfaces, TeleKyb can be easily extended to meet new requirements. The controller included in TeleKyb was proven to work well with completely different quadrotor UAVs. In the past, early versions of TeleKyb have been already used successfully for various applications [8], [13], [14].

With the release of the TeleKyb source code into a public ROS repository, we are hoping for contributing the sharing our knowledge with the robotics community, as well as receiving a valuable feedback on our work.

\section{A. Future Work}

Currently, we are working on the integration of a filter to allow for the use of TeleKyb with noisy low-frequency position data from, e.g., GPS or on-board visual state estimates.

\section{ACKNOWLEDGEMENTS}

Volker Grabe wishes to thank Dr. Davide Scaramuzza for hosting his visit at the University of Zurich in 2012/2013 and for providing the Nano+ quadrotor used in the experiments.

\section{REFERENCES}

[1] A. Makarenko, A. Brooks, and T. Kaupp, "On the benefits of making robotic software frameworks thin," in Workshop on Measures and Procedures for the Evaluation of Robot Architectures and Middleware at the 2007 IEEE/RSJ Int. Conf. on Intelligent Robots and Systems, San Diego, CA, Oct. 2007.
[2] A. Brooks, T. Kaupp, A. Makarenko, S. Williams, and A. Orebäck, "Orca: A component model and repository," in Software Engineering for Experimental Robotics, ser. Tracts in Advanced Robotics, D. Brugali, Ed. Springer, 2007, vol. 30, pp. 231-251.

[3] G. Metta, P. Fitzpatrick, and L. Natale, "YARP: Yet Another Robot Platform," International Journal of Advanced Robotic Systems, vol. 3, no. 1, pp. 43-48, 2006.

[4] M. Quigley, K. Conley, B. Gerkey, J. Faust, T. Foote, J. Leibs, R. Wheeler, and A. Y. Ng, "ROS: an open-source Robot Operating System," in Workshop on Open Source Software in Robotics at the 2009 IEEE Int. Conf. on Robotics and Automation, Kobe, Japan, May 2009.

[5] R. Simmons and D. James, Inter-Process Communication A Reference Manual. Carnegie Mellon University, 2011. [Online]. Available: http: / /www. cs. cmu.edu/îpc/

[6] J. Engel, J. Sturm, and D. Cremers, "Accurate figure flying with a quadrocopter using onboard visual and inertial sensing," in Workshop on Visual Control of Mobile Robots (ViCoMoR) at the 2012 IEEE/RSJ Int. Conf. on Intelligent Robots and Systems, Vilamoura, Portugal, Oct. 2012.

[7] M. Achtelik, M. Achtelik, S. Weiss, and R. Siegwart, "Onboard IMU and monocular vision based control for MAVs in unknown in- and outdoor environments," in 2011 IEEE Int. Conf. on Robotics and Automation, Shanghai, China, May 2011, pp. 3056-3063.

[8] A. Franchi, C. Secchi, M. Ryll, H. H. Bülthoff, and P. Robuffo Giordano, "Shared control: Balancing autonomy and human assistance with a group of quadrotor UAVs," IEEE Robotics \& Automation Magazine, Special Issue on Aerial Robotics and the Quadrotor Platform, vol. 19, no. 3, pp. 57-68, 2012.

[9] B. Hannaford and A. M. Okamura, "Haptics," in Springer Handbook of Robotics, B. Siciliano and O. Khatib, Eds. Springer, 2008, pp. 719-739.

[10] C. Masone, A. Franchi, H. H. Bülthoff, and P. Robuffo Giordano, "Interactive planning of persistent trajectories for human-assisted navigation of mobile robots," in 2012 IEEE/RSJ Int. Conf. on Intelligent Robots and Systems, Vilamoura, Portugal, Oct. 2012, pp. 2641-2648.

[11] M. Riedel, "Telekyb: A modular software framework for bilateral teleoperation scenarios and its applications in robotics research," Master Thesis, Eberhard Karls Universität Tübingen, 2012.

[12] J. Lächele, A. Franchi, H. H. Bülthoff, and P. Robuffo Giordano, "SwarmSimX: Real-time simulation environment for multi-robot systems," in 3rd Int. Conf. on Simulation, Modeling, and Programming for Autonomous Robots, Tsukuba, Japan, Nov. 2012.

[13] A. Franchi, C. Masone, V. Grabe, M. Ryll, H. H. Bülthoff, and P. Robuffo Giordano, "Modeling and control of UAV bearingformations with bilateral high-level steering," The International Journal of Robotics Research, Special Issue on 3D Exploration, Mapping, and Surveillance, vol. 31, no. 12, pp. 1504-1525, 2012.

[14] A. Franchi, C. Secchi, H. I. Son, H. H. Bülthoff, and P. Robuffo Giordano, "Bilateral teleoperation of groups of mobile robots with time-varying topology," IEEE Trans. on Robotics, vol. 28, no. 5, pp. 1019-1033, 2012. 